\title{
Using GC-MS for natural product analysis in Nigeria: Problems and prospects
}

\author{
B. O. Odjobo ${ }^{1}$, N. Ichoron ${ }^{2}$, N. P. Igoli ${ }^{3}, J$ O. Igoli $^{2 *}$ \\ ${ }^{1}$ BIODEC - National Biotechnology Development Agency (NABDA), Abuja Nigeria. \\ ${ }^{2}$ Department of Chemistry, University of Agriculture, P.M.B. 2373, Makurdi Nigeria \\ ${ }^{3}$ Centre for Food Technology and Research, Benue State University, P.M.B. 102119, Makurdi Nigeria \\ *Corresponding author: igolij@gmail.com; Phone 08130991308 \\ Received 22 May 2020; accepted 01 June 2020, published online 28 August 2020
}

\begin{abstract}
The absence or idle state of analytical equipment such as nuclear magnetic resonance spectroscopy, mass spectrometers and chromatographic techniques has constrained natural products research in Nigeria. Presently, gas chromatography-mass spectrometry has become the more easily available method in Nigeria for the identification of natural products in extracts. This method is sometimes fraught with nonreproducible results and the identification of non-natural or biosynthesized compounds. Several of the extracts analyzed are not volatile, some of the analysts are not competent and certified reference standards are hardly used. These coupled with poor library or database matches and data processing among others lead to the identification of compounds not recognizable from the extract or plants materials under study. This review discusses some of the problems involved in the use of this method for dereplication studies of plant extracts in Nigeria and makes some suggestions for improvement.
\end{abstract}

Key words: Dereplication, natural products, medicinal plants, extracts, GC-MS, spectroscopy

\section{Introduction}

The relevance of natural products and medicinal plants in drug discovery and identification of active molecules has been well reviewed [1-6]. The problem today is the slow pace of discovery of new active compounds from reportedly active medicinal plant extracts [7]. The identification of novel drug candidate molecules from forests, soils, oceans, fungi, bacteria or other microbial sources requires considerable investments in techniques, equipment, time and human resources [8]. While several reports of active extracts abound, they are not backed up by the isolation and characterization of their true active constituents. Rather efforts to determine the active constituents have ended up in the isolation or identification of well-known or previously isolated compounds $[18,19]$. One process of avoiding the waste of time and resources only to isolate previously known constituents is dereplication studies which enables the identification of known substances present in a mixture and enable focus on the discovery of new ones [8]. A range of dereplication methods are currently available to meet this challenge of improving natural product screening programs and thus distinguish novel entities from known natural compounds at an early stage $[8,20]$. The advent of hyphenated techniques such as GCMS, HPLC-MS, HPLC-NMR and HPLC-NMRMS has eased and enabled rapid compound separation and identification. They have also made possible metabolomics and dereplication studies and the identification of new metabolites and drug molecules. However, basic chromatographic and separation techniques, NMR, MS and HRMS are absent in Nigeria hence the most common practice in Nigeria now is GC-MS analysis of crude extracts. This method, GC-MS analysis [23,24] ideally separates the constituents of the extracts (which must be volatile or semi-volatile) and simultaneously identifies them, facilitating dereplication [25], such that the need to tediously isolate and characterize the components, especially known compounds, is removed [37]. However, the method, as applied presently in Nigeria, is fraught with errors and a lot of unusual and non-natural or nonbiosynthesized compounds are identified. This paper examines some of the challenges in the use of GC-MS, problems of its application in Nigeria and makes suggestions for improvement to obtain better and credible results. 


\section{Challenges in the use of GC-MS for analysis}

\section{GC/MS database}

Compared to other types of chromatography and mass spectrometry hyphenated techniques, GCMS has a large number of databases, such as national institute of standards and technology (NIST), dictionary of natural products (DNP) and MZmine. However, many GC-MS peaks still cannot be resolved by the available commercial MS databases [19]. The structures of metabolites in plants are complex, and so a metabolomics-targeted database will be more appropriate. Many databases, such as human metabolomics database, plant metabolomics database, and species-specific metabolomics database, are being continuously established and improved [28-31].

\section{Thermal Degradation}

Multi-peak phenomena result from metabolites producing multiple peaks, and this can be caused by sample degradation, by-product formation and the introduction of exogenous contaminants. The multi-origination phenomenon means one peak has multiple origins or precursors. Thermal non-stable compounds are prone to be thermally degraded, resulting in multi-peak and multiorigination phenomena [32].

\section{Deconvolution}

The aim of deconvolution is to analyze overlapping or co-eluted peaks and obtain the MS peak of a single pure substance. Currently, there are only a few good deconvolution software such as AMDIS, and commercial software such as ChromaTOF and AnalyzerPro $[33,34]$.

\section{Poor Baseline}

Baseline position could change suddenly during the run. This usually results from a range or attenuation change. It can also be as a result of valve operations, if valves are switched during the run, septum leak, wander and drift may occur when a flow or temperature setting is changed, or a damaged column is used. If the system is not stabilized at the new conditions before starting a run, some baseline changes are to be expected. An erratic or wandering baseline or one that moves up and down could be due to contamination in career gas, injector or a leak in the system. If there is a leak at the detector end of the system, then retention times should be stable and only sensitivity may be reduced. A leak at the inlet end of the column will result in decreased sensitivity and delayed retention times. Hence baseline problems could arise from several sources, some of which are electronic or mechanical failure, contamination in critical areas, such as detectors, incorrect or inappropriate setpoints; leaks, column or septum bleed, and other chromatographic difficulties $[32,35,36,38]$.

\section{Noise}

Noise occurs as rapid vertical baseline fluctuations, broadening of the baseline and giving it a hairy appearance. Noise is quite distinct from the random, large spiking peaks. Small amounts of noise are present in most detectors. At high attenuation, noise may be invisible but appears as attenuation is decreased. If noise appears suddenly on a previously clean baseline then attenuation should be reduced but if the noise decreases when the inlet temperature is lowered, then this is the likely cause of the noise. Noise could also appear from contaminated carrier gases. Similarly, air currents from a fan or air conditioner blowing across the top of the instrument may interfere with gas exiting from the detector and a contaminated detector results in noise. When noise increases gradually to an unacceptable level, it indicates a gradual buildup of the noise source, rather than an abrupt change $[39,40]$.

\section{Common problems in using GC-MS for natural products dereplication studies in Nigeria}

The problems associated with using GC-MS in Nigeria for natural products analysis include poor quality of extracts and in many cases the same column is used for all analysis. An overused column with residual components or fixated impurities could result in a high or poor baseline and a lot of noise from impurities in the 
spectrum. Some of the compound libraries for matching and comparison are not well extended to cover all classes of compounds or may not be regularly updated. Since most of the assays are on the "active" extracts, these extracts are usually methanol or aqueous extracts which are non-volatile. These extracts contain polymeric (polysaccharides or starch) and polar substances which may not be volatile under run temperatures (80-300 ${ }^{\circ} \mathrm{C}$ ). Some low melting point compounds or compounds with poor stability may decompose or undergo thermal degradation and rearrangements under the conditions. Plasticizers from solvents such as diisobutylphthalates are usually detected as they come from poorly purified solvents or industrial grade solvents transferred into Winchester bottles as analytical grade solvents. Cases of petrol being transferred into Winchester bottles as petroleum ether have been found. The lack of references and standards in their GC-MS runs (spiking- addition of internal standards for quantification) makes the spectra to be absolute rather than referenced spectra thus jeopardizing correlation of identified compounds to expected or previously isolated compounds.

Skilled personnel to handle the instrument are lacking as most organizations in Nigeria who acquire this equipment never put in place adequate training and retraining plan for the technicians. Also, many of the schools do not educate the students enough on the basics of GC-MS hence, many of the postgraduate and undergraduate students who are in most instances asked by their supervisors to use the method have little or no idea about it. And they in turn submit poorly prepared samples for GCMS analysis. Similarity index is usually low in most cases as other compounds or derivatives compete with the components of interests, this can be overcome when proper extraction is done using standard protocols.

\section{Suggestions for improving the use of GC-MS for natural product studies in Nigeria}

Sample analysis using GC-MS technique has been a most promising research aim in Nigeria though the results hardly meet expectations or are of any significant scientific value.
Improvements in this aspect of research can be brought about by

(a) Sample preparation: Extracts should be cleaned or pre-fractionated using silica gel or alumina so as to have a freeflowing extract. If column chromatography is not desired, a simple small column cleaning should be used and all eluates collected in a single vial, evaporated free of the solvent and reconstituted for analysis.

(b) Recognition of trace compounds or signal molecules: It is often desirable to add a trace of a known compound or standard for each run. This will serve as a signal compound among the other compounds or metabolites. The correct identification and quantification of such a compound gives integrity to the spectrum. As much as possible, similarly or previously isolated or reported compounds from the plant should be identified and a private library created for the spectrum. The spectrum should be reproducible qualitatively and quantitatively. This can be checked by running a known mixture of reference compounds. This known mixture can be used to spike the extract samples and by comparison to the reference compounds, the quantities of the sample constituents can be rationalized.

(c) Methods validation should be conducted, when new methods or modified methods are used. Laboratories should participate in Inter -Laboratory Comparison Tests, this should be part of the Analytical lab policy.

(d) Where novel compounds or constituents are inferred, efforts should be made to isolate such constituents and the GC-MS repeated and coupled with high resolution mass spectrometry and other spectroscopic techniques, the novel compound(s) can only then be identified.

(e) Several blanks and controls should be run at the initial stage. Blanks could be run with the extraction solvents or solvent mixtures. Controls could be run 
with standards or known compounds and can be used as references to establish the integrity of the spectrum.

(f) A proper and detailed literature survey to identify all previously identified compounds either by GC-MS or any spectroscopic method should be carried out and such compounds should be confirmed to be in the database. Compounds that are not natural or biosynthesized should be discarded and removed from the results. Similarly, for impurities and compounds arising from the column such as silicon esters should be discarded and removed from the results, unless the compounds were derivatives in the first instance.

(g) Improved data processing using statistical approaches and chemometrics $[24,25]$ or deconvolution methods [26].

\section{References}

1. M. A. Qaraghuli, R. Abdullah, A. R.Alzahrani, K. Niwasabutra, M. A. Obeid, V.A. Ferro (2017). Where Traditional Drug Discovery Meets Modern Technology in the Quest for New Drugs. Annals of Pharmacology and Pharmaceutics. 2(11): 1061.

2. D. J. Newman, G. M. Cragg (2016). Natural Products as Sources of New Drugs from 1981 to 2014. Journal of Natural Products. 79(3): 629-61

3. R. Batista, A. De Jesus Silva Júnior, A.B. De Oliveira (2009). Plant-Derived Antimalarial Agents: New Leads and Efficient Phytomedicines. Part II. NonAlkaloidal Natural Products. Molecules. 14: $3037-3072$

4. D. J. Newman, G. M. Cragg (2007). Natural products as sources of new drugs over the last 25 years. Journal Natural Products 70(3): 461-77.

5. G.M. Cragg and D. J. Newman (2001). Natural product drug discovery in the next millennium. Pharmeutical Biology 39(1): 8-17.

6. G. A. Cordell (2000). Biodiversity and drug discovery- a symbiotic relationship. Phytochemistry. 55: 463480

7. A. G. Atanasov, B. Waltenberger, E. Pferschy-Wenzig, T. Linder, C. Wawrosch, P. Uhrin, V. Temml, L. Wang, S. Schwaiger, E. Heiss, J. M. Rollinger, D. Schuster, J. M. Breuss, V. Bochkov, M. D. Mihovilovic, B. Kopp, R. Bauer, V. M. Dirsch, and H. Stuppner (2015). Discovery and resupply of pharmacologically active plantderivednatural products: A review. Biotechnology Advances 33: 1582-1614

8. J. Hubert, J. M. Nuzzilard, J. H. Renault (2017). Dereplication strategies in natural products research: How many tools and methodologies behind the same concept. Pyhtochemistry Review. 16: 55-95.

9. J.N. Anowu (2015). Studies on Seeds of Dacryodes elulis: Isolation of Gallic Acid

and its Antimicrobial Properties. M.Sc. Thesis; Department of Chemistry, University of Agriculture, Makurdi. pp 28-31

10. J. D. Bade, A. N., Ahera, S. C., Mandal, D. P. Belsare, and S. C. Pal (2010).

Isolation and characterization of hydrolysable tannins from leaf of Terminalia bellirica Roxb., Journal of Pharmaceutical Research, 3: 24102414

11. T.Y. Dikko, E.M. Khan, A.T. TorAnyiin, J. V. Anyam, and A.U. Linus (2016). In-

vitro Antimicrobial Activity of Fruit Pulp Extracts of Azanza garckeana (F.Hoffm). Exell and Hillc. And Isolation of one of its active principles, Betulinic acid. British Journal of Pharmaceutical Research, 14(1):1-10.

12. A. I. Gray, J. O. Igoli, and E. RuAngelie (2012). Natural Products Isolation in Modern Drug Discovery Programs in: Satyajit D. S. and Lutfun N. (eds.) Methods in Molecular Biology, pp 515534. DOI 10.1007/978-1-61779-6241_20. 
13. Mann, M.O. Fatope and J.I. Okogun (2012). Isolation and Elucidation of Three

Triterpenoids and its Antimycobacterial Activity of Terminalia avicenioides. American Journal of Organic Chemistry, 2(2):14-20. DOI 10.5923/j.ajoc.201120202.03

14. T. Patnaik, R.K. Dey and P. Gouda (2007). Isolation of Triterpenoid Glycoside from

Bark of Terminalia arjuna using Chromatographic Technique and Investigation of Pharmacological Behavior upon Muscle Tissue. EJournal of Chemistry; 4(4): $474-479$

15. Ramesh, A.S., Christopher, J.C., Radhika, R. and Setty, C.R. (2012). Isolation,

Characterization and Cytotoxicity Study of Arjunolic Acid from Terminalia arjuna. Natural Products Research; 26(16): 1549-1552.

16. D. Satyajit, Z.L. Sarker and I.G. Alexander (2006). Methods in Biotechnology, Vol.

20, Natural Products Isolation, 2nd ed. Humana Press Inc., Totowa, New Jersey, U.S.A.

17. T. Tanaka, G.I. Nonaka and I. Nishioka (1986). Tannins and Related Compounds.

XLII: Isolation and Characterization of Four New Hydrolyzable Tannins, Terflavins $\mathrm{A}$ and $\mathrm{B}$, Tergallagin and Tercatain from the Leaves of Terminalia catappa L. Chemical and Pharmaceutical Bulletin, 34: 10391049.

18. S. Visht and S. Chaturvedi (2012). Isolation of Natural Products. Curremt Pharma

Research, 2(3): 584-599.

19. X. Qi, X. Chen, and Y. Wang (2014). Plant Metabolomics: Methods and Applications. Springer, Dordrecht, Netherland. p. 42.

20. J.R. Doroghazi, J.C. Albright, A.W. Goering, K. Ju, R.R. Haines, K.A. Tchalukov, D.P. Labeda, N. L. Kelleher and W.W. Metcalf (2014). A roadmap for natural product discovery based on large-scale genomics and metabolomics. Nature Chemical Biology. 10(11): 963-8.

21. U.Roessner, D.A. Dias. (2013). Metabolomics Tools for Natural Product Discovery-Methods and Protocols. Springer New York. p 313.

22. M.S. Alam and R.M. Harrison (2016). Recent advances in the application of 2dimensional gas chromatography with soft and hard ionization time-of-flight mass spectrometry in environmental analysis. Chemical Science 7: 39683977

23. Z.H. Syed and M. Khushnuma (2014). GC-MS: principle, technique and its application in food science. International Journal of Current Science.13E: 116-126.

24. N. F. Carnevale, A. C. Pilon, D.M. Selegato, R.T. Freire, H. Gu, D. Raftery, N.P. Lopes, I. Castro-Gamboa (2019). Dereplication of Natural Products Using GC-TOF Mass Spectrometry: Improved Metabolite Identification by Spectral Deconvolution Ratio Analysis. Frontiers in Molecular Biosciences 3: 59

25. Biancolillo A and Marini F. (2018). Chemometric Methods for Spectroscopy-Based Pharmaceutical Analysis. Frontiers in Chemistry ; 6:576. doi: 10.3389/fchem.2018.00576

26. $\mathrm{Vu}$ Dang $\mathrm{H}$ and Marini F. (2019). Editorial: Chemometrics-based Spectroscopy for Pharmaceutical and Biomedical Analysis. Frontiers in Chemistry. 7:153. doi: 10.3389/fchem.2019.00153

27. C. L. Zani and A.R. Carroll (2017). Database for Rapid Dereplication of Known Natural Products Using Data from MS and Fast NMR Experiments. Journal of Natural Products 80(6): 1758-1766.

https://doi.org/10.1021/acs.jnatprod.6b0 1093

28. A. Fukushima and M. Kusano (2013). Recent progress in the development of 
metabolome databases for plant systems biology. Frontiers in Plant Science, 4:73

29. D. B. Kell and S. G. Oliver. (2016). The metabolome 18 years on: a concept comes of age. Metabolomics 12: 148.

30. J. L. Griffin and A. W. Nicholls (2006). Metabolomics as a functional genomic tool for understanding lipid dysfunction in diabetes, obesity and related disorders. Pharmacogenomics, 7(7): 1095 - $107 . \quad$ DOI: $102217 / 14622416.7 .7 .1095$

31. O. Fiehn (2001). Combining genomics, metabolome analysis, and biochemical modelling to understand metabolic networks. Comparative and functional genomics 2, 155-168.

32. D. Rood (1996). Gas Chromatography Problem Solving and Troubleshooting. Journal of Chromatographic Science. 34: 305-306.

33. O.D. Ogundele, S.O. Thompson, O. Jayeola Johnson and O. Olansileadedamola (2019). Gas Chromatography-Mass Spectrometry analysis of chromatographic fractions of Nigerian bitumen International Journal of Current Research in Applied Chemistry \& Chemical Engineering. 3: 32-38

34. X. Du and S. H. Zeisel (2013). Spectral deconvolution for gas chromatography mass spectrometry - based metabolomics: Current status and future perspectives. Computational and structural biotechnology, 4(5): 1-11.
35. D. Rood (1998). Gas Chromatography Problem Solving and Troubleshooting. Journal of Chromatographic Science. 36: $379-380$.

36. D. J. Morgan (2014). 5 Ways to approach baseline issues. Accessed on $15^{\text {th }} \quad$ April, 2020 from https://www.chromatography today.com/news/gc-mdgc/32/breakingnews/5-ways-to-approach-baselineissues/31524

37. F. Douglas (2019). GC/MS Analysis. Accessed on 13th April 2020 from http://www.scientific.org/tutorials/articl es/gcms.html

38. D. E. Matthews and J. M. Hayes (1976). Systematic errors in gas chromatography-mass spectrometry isotope ratio measurements. Analytical Chemistry 48(9): 1375-1382

39. M. B. Wilson B. B. Barnes and P.G. Boswell (2014). What Experimental Factors Influence the Accuracy of Retention Projections in Gas Chromatography-Mass Spectrometry? Journal of Chromatography A. 19(1373): 179-189.

40. H. Perry (2014). 8 Common Gas Chromatography Mistakes. Accessed on 15th April, 2020 from https://www.chromatographytoday.com/ news/gc-mdgc/32/breaking-news/8common-gas-chromatographymistakes/31345 